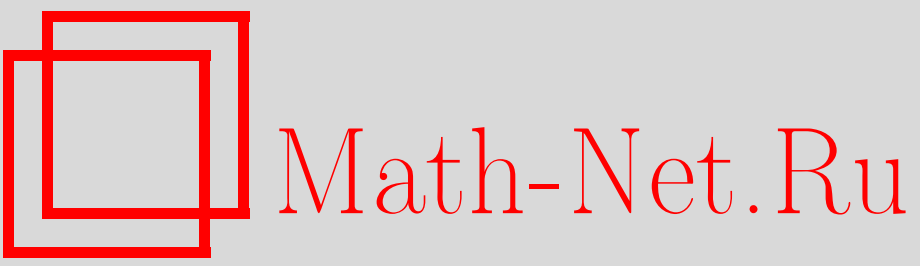

А. Н. Козлов, Численная модель инжекции потока плазмы в магнитное поле соленоида, Матем. моделирование, 2020, том 32, номер 5, 3-20

DOI: https://doi.org/10.20948/mm-2020-05-01

Использование Общероссийского математического портала Math-Net.Ru подразумевает, что вы прочитали и согласны с пользовательским соглашением

http://www.mathnet.ru/rus/agreement

Параметры загрузки:

IP : 54.164 .48 .24

26 апреля 2023 г., $17: 25: 38$ 


\title{
ЧИСЛЕННАЯ МОДЕЛЬ ИНЖЕКЦИИ ПОТОКА ПЛАЗМЫ В МАГНИТНОЕ ПОЛЕ СОЛЕНОИДА
}

\author{
(C) 2020 г. А.Н. Козлов \\ ${ }^{1}$ Институт прикладной математики им. М.В. Келдыша РАН \\ ${ }^{2}$ МГУ им. М.В. Ломоносова, механико-математический факультет \\ andrey-n-kozlov@mail.ru
}

Исследование выполнено за счет гранта Российского научного фонда (проект № 16-11-10278-П).

DOI: $10.20948 / \mathrm{mm}-2020-05-01$

Рассмотрена инжекция потока плазмы, генерируемой с помощью квазистационарного плазменного ускорителя, в область с магнитным полем, создаваемым рядом кольцевых токонесущих проводников, образующих соленоид. Численные исследования проведены на основе системы МГД уравнений, представленных в терминах векторного потенциала магнитного поля, с учетом электропроводности, теплопроводности и переноса излучения при условии, что плазменный ускоритель и кольцевые проводники расположены на одной оси. Показано, что небольшие по величине токи в кольцевых проводниках создают магнитное поле внутри соленоида, которое не оказывает значительного влияния на основной поток, одновременно изолируя его от катушек с током в результате образования слоя разреженной плазмы, отделяющего высокоскоростной поток плотной плазмы. Расчеты МГД задачи показали также, что течение плазмы в пределах соленоида не сопровождается увеличением температуры среды.

Ключевые слова: модель магнитной газодинамики, течения плазмы, плазменный ускоритель, кольцевые проводники с током.

\section{NUMERICAL MODEL OF PLASMA FLOW INJECTION IN SOLENOID MAGNETIC FIELD}

\author{
A.N. Kozlov ${ }^{1,2}$ \\ ${ }^{1}$ Keldysh Institute of Applied Mathematics of RAS \\ ${ }^{2}$ Lomonosov Moscow State University, Faculty of Mechanics and Mathematics
}

Injection of plasma flow generated by the quasi-stationary plasma accelerator into a region with a magnetic field created by the series of ring current-carrying conductors form- 
ing a solenoid is considered. Numerical studies were carried out on the basis of the set of the MHD equations represented in terms of the vector potential of the magnetic field, taking into account electrical conductivity, thermal conductivity and radiation transport, provided that the plasma accelerator and ring conductors are located on the same axis. It is shown that small currents in ring conductors create a magnetic field inside the solenoid, which does not significantly affect the main stream, while simultaneously isolating it from the current coils as a result of the formation of the rarefied plasma layer separating high-speed flow of dense plasma. Calculations of the MHD problem also showed that the plasma flow within the solenoid is not accompanied by an increase in the temperature of the medium.

Key words: magnetogasdynamics model, plasma flows, plasma accelerator, ring conductors with a current.

\section{1. Введение}

Квазистационарные плазменные ускорители (КСПУ) являются многофункциональными системами, предназначенными для различных технологических приложений и термоядерных исследований, в том числе по программе ИТЭР, а также представляют интерес для разработки перспективных мощных электрореактивных плазменных двигателей как системы проточного типа, в которых на входе непрерывно подается газ. Исследование высокоскоростных потоков плазмы в КСПУ направлено, в частности, на решение задачи инжекции в термоядерные установки. Использование плазменных ускорителей в качестве инжекторов в магнитные системы для удержания плазмы предполагает согласованное поступление потоков из ускорителей в магнитные ловушки. Взаимодействие потока плазмы с магнитным полем, создаваемым проводниками с током в системах для удержания плазмы, может быть рассмотрено на основе магнитной газодинамики или МГД моделей при наличии трехкомпонентного магнитного поля. Инжекция потока плазмы в магнитное поле одного кольцевого проводника с током рассмотрена в [1]. Численное исследование инжекции плазмы в ряд кольцевых проводников, расположенных на определенном расстоянии друг от друга, представлено в [2]. Случай плотно расположенных катушек с током, образующих соленоид, рассмотрен в данной работе.

Теоретические, численные и экспериментальные работы (см., например, [2-18]) по изучению физических процессов в КСПУ и магнитоплазменных компрессорах (МПК) формируют одно из направлений в вычислительной плазмодинамике и физике плазмы, охватывая широкий круг задач, в том числе в области радиационной плазмодинамики и управляемого термоядерного синтеза (УТС). С помощью МГД моделей различного уровня сложности исследуется динамика плазмы в КСПУ при наличии дополнительного 
продольного магнитного поля [9-13], изучаются приэлектродные процессы, обусловленные эффектом Холла $[11,12]$, а также компрессионные потоки [3-5,14], динамика примесей [15] и перенос излучения [16]. Разработаны различные численные модели для изучения процесса ионизации в канале плазменного ускорителя [16-18].

Классические плазменные ускорители состоят из двух коаксиальных электродов, подключенных к электрической цепи. В простейших системах процессы происходят при наличии только азимутальной компоненты магнитного поля, которое генерируется электрическим током, проходящим вдоль внутреннего электрода. Радиальный плазменный ток, протекающий между электродами, и азимутальное магнитное поле обеспечивают ускорение плазмы благодаря силе Ампера $\frac{1}{c} \mathbf{j} \times \mathbf{H}$, где $\mathbf{j}-$ ток в плазме. КСПУ в полноблочном исполнении является двухступенчатой системой. Первая ступень включает ряд малых плазменных ускорителей, в которых происходят ионизация поступающего газа и предварительное ускорение плазмы. Вторая ступень представляет собой ускоритель больших размеров, подключенный к отдельной цепи питания, и обеспечивает окончательное ускорение плазмы.

Плазменные ускорители могут быть использованы для наполнения различных установок по удержанию плазмы, включая ловушки «Галатеи» с левитирующими проводниками, погруженными в плазму (см., например, [1921]) и многопробочные ловушки [22] для разреженной плазмы. В [2] показано, что инжекция высокоскоростных потоков достаточно плотной плазмы, генерируемой КСПУ, в ряд кольцевых проводников с током может оказаться более эффективным способом достижения конечного результата в решении проблемы УТС.

Исследования, проводимые для различных магнитных ловушек, связаны, в частности, с изучением взаимодействия потока плазмы с магнитным полем кольцевых проводников с током. Один из способов включения в модель и расчетную область кольцевых проводников был рассмотрен ранее в упрощенной модели (см., например, [23]). В статьях [1,2], а также в данной работе, использован иной способ включения в модель кольцевых токонесущих проводников для исследования взаимодействия потока плазмы из КСПУ с магнитным полем, создаваемым проводниками.

\section{2. МГД уравнения в терминах векторного потенциала магнитного поля}

Численные исследования процессов для достаточно плотной плазмы основаны на МГД моделях (см., например, [3,5,24-28]). Изучение процессов в КСПУ проводится с помощью различных модификаций МГД уравнений, 
включая одножидкостные модели (см., например, [3-5,10-14]) и двухжидкостные модели с учетом эффекта Холла $[9,11,12]$. В последние годы для изучения процессов в КСПУ разработана модель радиационной магнитной газодинамики, когда МГД задача решается одновременно с уравнением переноса излучения [16]. Учет тех или иных диссипативных факторов и различных процессов обусловлен деталями постановок задач.

В настоящей работе с учетом экспериментальных данных рассматривается осесимметричный поток плазмы в канале между двумя коаксиальными профилированными электродами, инжектируемый в магнитное поле соленоида при условии, что внешняя граница плазменного объема внутри соленоида ограничена изолятором, являющимся продолжением цилиндрической поверхности внешнего электрода ускорителя. В задаче участвуют все три компоненты магнитного поля $\mathbf{H}=\left(H_{z}, H_{r}, H_{\varphi}\right)$ при наличии продольного поля, создаваемого кольцевыми токонесущими проводниками, образующими соленоид, а также при наличии азимутального поля в профилированной части ускорителя и на выходе из него. Продольное поле приводит к вращению плазмы. Соответственно в задаче участвуют три компоненты скорости $\mathbf{V}=\left(V_{z}, V_{r}, V_{\varphi}\right)$. Для квазинейтральной среды $n_{i}=n_{e}=n$ пренебрегаем инерцией электронов $\left(m_{e}<<m_{i}\right)$ и током смещения. В соответствии с экспериментальными данными о КСПУ рассмотрим случай однотемпературной смеси $T_{i} \approx T_{e}=T$. Исследование проведено в рамках одножидкостной модели $\left(\mathbf{V}_{e}=\mathbf{V}_{i}=\mathbf{V}\right)$. Постановка задачи включает уравнения радиационной магнитной газодинамики с учетом конечной проводимости среды, теплопроводности и переноса излучения

$$
\begin{aligned}
& \frac{\partial \rho}{\partial t}+\operatorname{div} \rho \mathbf{V}=0, \quad \rho \frac{d \mathbf{V}}{d t}+\nabla P=\frac{1}{c}[\mathbf{j}, \mathbf{H}], \\
& \frac{\partial}{\partial t}(\rho \varepsilon)+\operatorname{div}(\rho \varepsilon \mathbf{V})+P \operatorname{div} \mathbf{V}=\frac{\mathbf{j}^{2}}{\sigma}-\operatorname{div} \mathbf{q}-\operatorname{div} \mathbf{W}, \\
& \frac{\partial \mathbf{H}}{\partial t}=\operatorname{rot}[\mathbf{V}, \mathbf{H}]-c \operatorname{rot} \frac{\mathbf{j}}{\sigma}, \quad \quad \mathbf{j}=\frac{c}{4 \pi} \operatorname{rot} \mathbf{H}, \\
& \frac{d}{d t}=\frac{\partial}{\partial t}+(\mathbf{V}, \nabla), \quad \rho=m_{i} n, \quad P=P_{i}+P_{e}=2 k_{B} n T, \quad \varepsilon=2 c_{v} T .
\end{aligned}
$$

Переменные в системе МГД уравнений имеют обычный смысл. Электропроводность среды равна $\sigma=e^{2} n_{e} \tau_{e} / m_{e}$, где $\tau_{e}$ - характерное время столкновений электронов с ионами. Вектор $\mathbf{q}=-\kappa \nabla T$ отвечает потоку теп- 
ла, где к - коэффициент теплопроводности. Вязкостью компонент плазмы можно пренебречь в силу малого влияния соответствующих диссипативных факторов.

В уравнении для внутренней энергии плотность потока энергии излучения $\mathbf{W}$, а также плотность энергии излучения $U$, определяются через интенсивность излучения $I_{v}(\mathbf{r}, \boldsymbol{\Omega})$ с частотой $v$ вдоль выбранного направления телесного угла $\boldsymbol{\Omega}$ в точке с координатой $\mathbf{r}$

$$
U(\mathbf{r})=\frac{1}{c} \int_{0}^{\infty} \int_{0}^{4 \pi} I_{v}(\mathbf{r}, \mathbf{\Omega}) d \Omega d v, \quad \mathbf{W}(\mathbf{r})=\int_{0}^{\infty} \int_{0}^{4 \pi} I_{v}(\mathbf{r}, \mathbf{\Omega}) \boldsymbol{\Omega} d \mathbf{\Omega} d v .
$$

Плотность энергии излучения $U$ можно не учитывать в МГД уравнениях, так как $U<<\rho \varepsilon$. В то же время поток энергии излучения $W \sim c U$ может играть определенную роль в перераспределении энергии. Поскольку поле излучения мгновенно подстраивается под распределение параметров потока, можно ограничиться решением стационарного уравнения переноса излучения

$$
\boldsymbol{\Omega} \cdot \nabla I_{v}(\mathbf{r}, \boldsymbol{\Omega})=\eta_{v}(\mathbf{r})-\kappa_{v}(\mathbf{r}) \cdot I_{v}(\mathbf{r}, \mathbf{\Omega}) .
$$

В уравнении (3) коэффициент поглощения $\kappa_{v}(\mathbf{r})$ и излучательная способность $\eta_{v}(\mathbf{r})$ зависят от состояния среды, ее плотности и температуры, а также спектрального параметра $v$, связанного с энергией фотона $h v$ (см., например, [28-33]). Данные коэффициенты складываются из трех частей, отвечающих поглощению и излучению в линиях, фотоионизации и фоторекомбинации, а также рассеянию. Более детально постановка задачи о переносе излучения в потоках плазмы и ионизующегося газа в КСПУ, а также формулы для определения коэффициента поглощения и излучательной способности, изложены в $[16,34,35]$. В целом влияние переноса излучения незначительно для исследуемых течений плазмы.

В численной модели уравнения (1) используются в безразмерной форме. В качестве единиц измерения возьмем длину канала $L$, а также характерные значения концентрации или плотности $n_{o}\left(\rho_{o}=m_{i} n_{o}\right)$, температуры $T_{o}$ и азимутальной составляющей магнитного поля на входе в канал $H_{o}=$ $=2 J_{p} / c R_{o}$, где $R_{o}$ - характерный радиус, $J_{p}$ - разрядный ток в системе. С помощью данных величин формируются единицы скорости $V_{o}=H_{o} / \sqrt{4 \pi \rho_{o}}$, времени $t_{o}=L / V_{o}$, тока в плазме $j_{o}=c H_{o} / 4 \pi L$, а также единица измерения азимутальной компоненты векторного потенциала магнитного поля 
$A_{o}=H_{o} L$, используемого в модели. Безразмерными параметрами модели являются отношение характерного газового давления к магнитному $\beta=$ $=8 \pi P_{o} / H_{o}{ }^{2}\left(P_{o}=2 k n_{o} T_{o}\right)$, магнитная вязкость $v=1 / \operatorname{Re}_{m}=c^{2} / 4 \pi L V_{o} \sigma$, которая обратно пропорциональна магнитному числу Рейнольдса $\operatorname{Re}_{m}=\sigma_{o} T^{3 / 2}$, a также безразмерное значение коэффициента теплопроводности $\tilde{\kappa}$. Переход к безразмерному значению плотности потока энергии излучения $\mathbf{W}$ в (1) и (2) осуществляется с помощью следующей единицы измерения: $W_{o}=$ $=V_{o} H_{o}^{2} / 4 \pi$.

Важным фактором в МГД моделях является сохранение свойства соленоидальности магнитного поля. Для осесимметричных течений плазмы $(\partial / \partial \varphi=0)$ можно использовать векторный потенциал $\mathbf{A}$ магнитного поля $\mathbf{H}=\operatorname{rot} \mathbf{A}$ так, что

$$
H_{r}=-\frac{\partial A_{\varphi}}{\partial z}, \quad H_{z}=\frac{1}{r} \frac{\partial\left(r A_{\varphi}\right)}{\partial r},
$$

где $A_{\varphi}$ - азимутальная компонента векторного потенциала А. При условии аксиальной симметрии соотношения (4) обеспечивают точное выполнение уравнения $\operatorname{divH}=0$. В осесимметричном случае систему МГД уравнений (1) можно записать в безразмерном виде в терминах $A_{\varphi}$-компоненты векторного потенциала и $H_{\varphi}$-компоненты магнитного поля:

$$
\begin{aligned}
& \frac{\partial \rho}{\partial t}+\frac{\partial\left(\rho V_{z}\right)}{\partial z}+\frac{1}{r} \frac{\partial\left(r \rho V_{r}\right)}{\partial r}=0 \\
& \frac{\partial\left(\rho V_{z}\right)}{\partial t}+\frac{\partial}{\partial z}\left(\rho V_{z}^{2}+P+\frac{H_{\varphi}^{2}}{2}\right)+\frac{1}{r} \frac{\partial}{\partial r}\left(r \rho V_{z} V_{r}\right)=j_{\varphi} \frac{\partial A_{\phi}}{\partial z} \\
& \frac{\partial\left(\rho V_{r}\right)}{\partial t}+\frac{\partial}{\partial z}\left(\rho V_{z} V_{r}\right)+\frac{1}{r} \frac{\partial}{\partial r}\left[r\left(\rho V_{r}^{2}+P+\frac{H_{\varphi}^{2}}{2}\right)\right]= \\
& =\frac{1}{r}\left(\rho V_{\varphi}^{2}+P-\frac{H_{\varphi}^{2}}{2}\right)+\frac{j_{\varphi}}{r} \frac{\partial\left(r A_{\varphi}\right)}{\partial r}, \\
& \frac{\partial D_{\varphi}}{\partial t}+\frac{\partial\left(V_{z} D_{\varphi}\right)}{\partial z}+\frac{1}{r} \frac{\partial\left(r V_{r} D_{\varphi}\right)}{\partial r}=\frac{\partial\left(r A_{\varphi}\right)}{\partial r} \frac{\partial H_{\varphi}}{\partial z}-\frac{\partial A_{\varphi}}{\partial z} \frac{\partial\left(r H_{\varphi}\right)}{\partial r} \\
& \frac{\partial(\rho \varepsilon)}{\partial t}+\frac{\partial\left(\rho \varepsilon V_{z}\right)}{\partial z}+\frac{1}{r} \frac{\partial\left(r \rho \varepsilon V_{r}\right)}{\partial r}=-P\left(\frac{\partial V_{z}}{\partial z}+\frac{1}{r} \frac{\partial\left(r V_{r}\right)}{\partial r}\right)-\left(\frac{\partial W_{z}}{\partial z}+\frac{1}{r} \frac{\partial\left(r W_{r}\right)}{\partial r}\right)+
\end{aligned}
$$




$$
\begin{aligned}
& +v\left\{\left[\frac{1}{r} \frac{\partial\left(r H_{\varphi}\right)}{\partial r}\right]^{2}+\left(\frac{\partial H_{\varphi}}{\partial z}\right)^{2}+j_{\varphi}^{2}\right\}-\left(\frac{\partial q_{z}}{\partial z}+\frac{1}{r} \frac{\partial\left(r q_{r}\right)}{\partial r}\right), \\
& \frac{\partial H_{\varphi}}{\partial t}+\frac{\partial\left(V_{z} H_{\varphi}\right)}{\partial z}+\frac{\partial\left(V_{r} H_{\varphi}\right)}{\partial r}=\frac{\partial\left(r A_{\varphi}\right)}{\partial r} \frac{\partial}{\partial z}\left(\frac{V_{\varphi}}{r}\right)-r \frac{\partial A_{\varphi}}{\partial z} \frac{\partial}{\partial r}\left(\frac{V_{\varphi}}{r}\right)+ \\
& +\frac{\partial}{\partial r}\left[\frac{v}{r} \frac{\partial\left(r H_{\varphi}\right)}{\partial r}\right]+\frac{\partial}{\partial z}\left(v \frac{\partial H_{\varphi}}{\partial z}\right), \\
& \frac{\partial A_{\varphi}}{\partial t}+V_{z} \frac{\partial A_{\varphi}}{\partial z}+\frac{V_{r}}{r} \frac{\partial\left(r A_{\varphi}\right)}{\partial r}=v\left(\Delta A_{\varphi}-\frac{A_{\varphi}}{r^{2}}\right)
\end{aligned}
$$

$j_{\varphi}=\frac{\partial H_{r}}{\partial z}-\frac{\partial H_{z}}{\partial r}=-\Delta A_{\varphi}+\frac{A_{\varphi}}{r^{2}}-$ азимутальный ток, $D_{\varphi}=r \rho V_{\varphi}, \varepsilon=\beta T /(\gamma-1)$, $\Delta A_{\varphi}=\frac{\partial^{2} A_{\varphi}}{\partial z^{2}}+\frac{1}{r} \frac{\partial}{\partial r}\left(r \frac{\partial A_{\varphi}}{\partial r}\right), P=\beta \rho T$. Уравнения (5) содержат семь независимых переменных - $\rho, T, V_{z}, V_{r}, V_{\phi}, H_{\phi}, A_{\phi}$.

\section{3. Постановка задачи}

Уравнения, безразмерные параметры, отвечающие законам подобия, и граничные условия определяют решение задачи и динамику потока плазмы. Численное интегрирование уравнений (5) ведется в области переменных $(z, r)$, соответствующей форме канала, изображенного на рис.1.

Во входном сечении канала ускорителя при $z=0$ полагаем, что плазма подается с известными значениями плотности $\rho(r)=f_{1}(r)$ и температуры $T(r)=f_{2}(r)$. Если не рассматривается дополнительное уравнение электрической цепи, то полагаем, что ток поддерживается постоянным и поступает в систему только через электроды, т.е. $j_{z}=0$ при $z=0$ или $r H_{\varphi}=r_{o}=$ $=$ const $\left(r_{o}=R_{o} / L\right)$. Дозвуковая подача плазмы осуществляется вдоль определенного направления, например, вдоль координатных линий. Эти стандартные граничные условия используются для численных моделей динамики плазмы в каналах в отсутствии продольного магнитного поля. В противном случае необходимы граничные условия для переменных $V_{\varphi}$ и $A_{\varphi}$ на входе в канал, рассмотренные, в частности, в [38].

Граничные условия на электродах $r=r_{\kappa}(z)$ и $r=r_{a}(z)$, формирующих стенки канала ускорителя, отвечают их эквипотенциальности и непроницаемости поверхности для плазмы. Соответственно на поверхности электродов имеем следующие условия: 
$E_{\tau}=0, \quad V_{n}=0$.

При наличии продольного магнитного поля в КСПУ необходимо дополнительное соотношение, связывающее компоненты магнитного поля на границе плазма-проводник [38].

a)

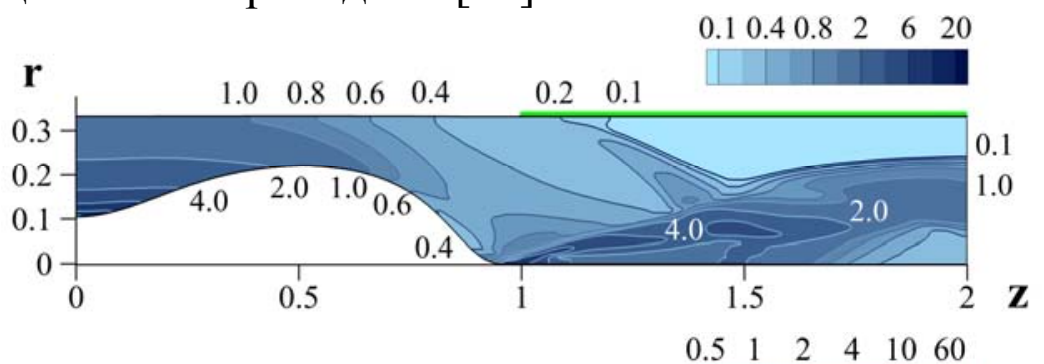

б)

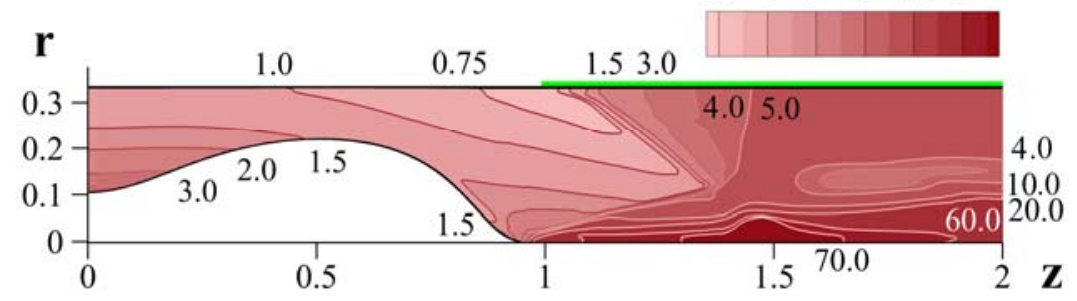

B)

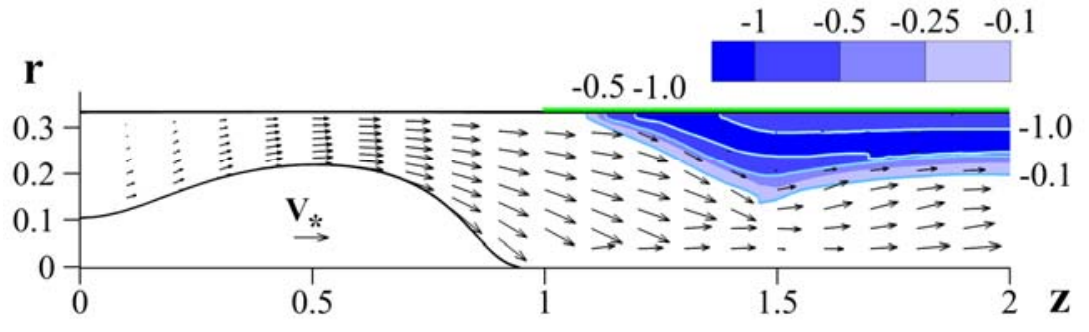

г)

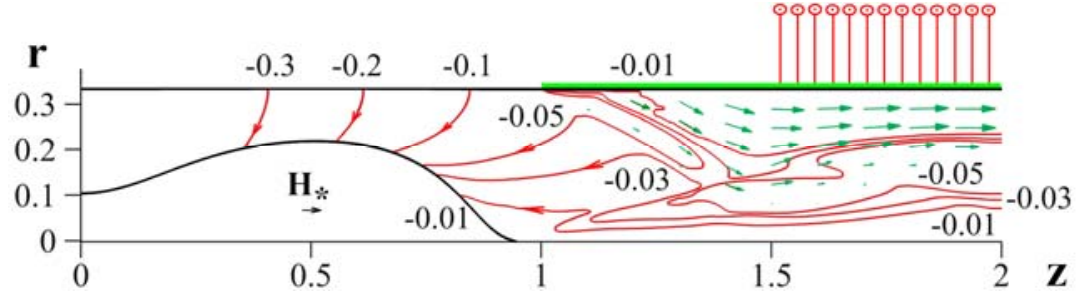

Рис.1. Распределения а) плотности, б) температуры, в) векторного поля скоростей и азимутальной скорости, г) плазменного тока и векторного магнитного поля при взаимодействии потока с магнитным полем соленоида.

В отсутствие продольного магнитного поля и при наличии единственной азимутальной компоненты магнитного поля в канале ускорителя в его наиболее узкой профилированной части происходит переход скорости потока через скорость быстрой магнитозвуковой волны или скорость сигнала [3], равную $C_{S}=\sqrt{C_{g}^{2}+C_{A}^{2}}$, где $C_{g}^{2}=\gamma P / \rho$ и $C_{A}^{2}=H^{2} / 4 \pi \rho$. В профилированном канале формируется трансзвуковое течение, и на выходе из ускорителя имеем сверхзвуковой поток плазмы.

Изолятор является границей между объемом плазмы и вакуумным магнитным полем, создаваемым кольцевыми проводниками с током. Использо- 
вание изолятора в модели дает возможность отказаться от расчетов обтекания плазмой отдельных проводников, что не является актуальной задачей, поскольку исследование направлено на изучение влияния магнитного поля соленоида на высокоскоростные потоки плазмы. Изолятор, отмеченный на рис.1, при $z>1$ обладает известными свойствами. Граничные условия на его поверхности имеют следующий вид:

$$
V_{n}=0, \quad j_{n}=0, \quad A_{\varphi}=A(z) .
$$

Значения азимутальной компоненты векторного потенциала $A_{\varphi}$ и функции магнитного потока $\psi=r A_{\varphi}$ на изоляторе следует задать. Функция $A(z)$ на поверхности изолятора определяется в результате решения задачи о вакуумном магнитном поле, создаваемом системой проводников.

Чтобы вычислить значения функции $A_{\varphi}=A(z)$ на изоляторе, в данном случае цилиндрической поверхности, сопряженной с каналом ускорителя и ограничивающей плазменный объем, следует обратиться к соотношениям, определяющим вакуумное магнитное поле, создаваемое кольцевыми проводниками (см., например, [24]).

В безразмерных переменных для одного кольцевого проводника с током $J_{\kappa}$ азимутальная компонента векторного потенциала равна

$$
A_{\varphi}^{\kappa}=2 \frac{J_{\kappa}}{J_{p}} \frac{R_{\kappa}}{F} \sqrt{\frac{R_{\kappa}}{r}}\left[\left(1-\frac{1}{2} F^{2}\right) G-U\right],
$$

где $G$ и $U$ - полные эллиптические интегралы 1-го и 2-го рода:

$G=\int_{0}^{\pi / 2} \frac{d \theta}{\sqrt{1-F^{2} \sin ^{2} \vartheta}}, \quad U=\int_{0}^{\pi / 2} \sqrt{1-F^{2} \sin ^{2} \vartheta} d \theta, \quad F^{2}=\frac{4 r R_{k}}{\left(R_{k}+r\right)^{2}+\left(z-z_{k}\right)^{2}}$, $R_{\kappa}$ - радиус кольца с током, $z_{\kappa}$ - положение кольца с током в цилиндрической системе координат. Значение $z=0$ отвечает входу в канал ускорителя. В силу принципа суперпозиции полей, справедливого и для магнитного поля, в случае нескольких проводников с индексом $k=1, \ldots . ., K$ суммарное значение азимутальной компоненты векторного потенциала равно $A_{\phi}=\sum_{k=1}^{K} A_{\phi}^{k}$.

Численному решению уравнений (5) с граничными условиями (6) и (7) предшествует отображение исходной расчетной области на прямоугольник в плоскости координат $(y, z)$ с помощью соотношения 


$$
r=(1-y) r_{\kappa}(z)+y r_{a}(z) .
$$

Расчет гиперболической части МГД уравнений основан на разностной схеме с коррекцией потоков [36]. Для учета магнитной вязкости и теплопроводности используется потоковый вариант метода прогонки [37]. Течения плазмы рассчитываются до установления в процессе численного решения исходной нестационарной МГД задачи. Алгоритм численного решения задачи о переносе излучения на основе метода длинных характеристик изложен в $[16,30,35]$.

\section{4. Результаты численных экспериментов}

Геометрия расчетной области, представленная на рис.1, отвечает численным исследованиям инжекции потока плазмы, генерируемой в КСПУ, в ряд близко расположенных токонесущих кольцевых проводников, образующих соленоид. Профиль канала ускорителя при $0 \leq z \leq 1$ отвечает аналитическим исследованиям двумерных течений плазмы $[9,10]$. В аналитической модели в приближении холодной плазмы плотность на входе в канал меняется по закону $\rho(z=0, r)=r_{o}^{2} / r^{2}$. Предполагая изэнтропичность поступающей плазмы $\left(S=c_{v} \ln P / \rho^{\gamma}=\mathrm{const}\right)$, имеем $T=\rho^{\gamma-1}$ при $z=0$. В расчетах считаем, что плотность и температура меняются на входе по указанным законам.

Кольцевые проводники с током при $1.5 \leq z \leq 2$ расположены вне расчетной области (см. рис.1г), что позволяет избежать моделирования обтекания плазмой отдельных проводников. На цилиндрической поверхности изолятора, являющегося продолжением внешнего цилиндрического электрода при $y=1$ и $1 \leq z \leq 2$, заданы значения функции магнитного потока $\psi=r A_{\varphi}$, отвечающей азимутальной компоненте векторного потенциала магнитного поля. Значения функции $\psi$ при $1 \leq z \leq 1.5$, определенные с помощью соотношения (8) и представленные на рис.2, соответствуют току в кольцевом проводнике, равному $J_{\kappa}=0.2 \cdot J_{p}$. При этом кольцо с током расположено в плоскости $z=z_{\kappa}=1.5$ и его радиус равен $R_{\kappa}=1.5 \cdot r_{o}$, где $r_{o}=R_{o} / L=0.33$. Постоянное значение функции $\psi$ при $y=1$ и $1.5 \leq z \leq 2$ соответствует магнитному полю соленоида на внешней границе расчетной области или поверхности изолятора, отделяющей объем плазмы от вакуумного магнитного поля между изолятором и кольцевыми проводниками.

Рис.1 иллюстрирует динамику установившегося потока плазмы из КСПУ, взаимодействующего с магнитным полем соленоида. Изолятор, на котором заданы значения $\psi=r A_{\varphi}$, дополнительно отмечен на внешней гра- 
нице расчетной области при $1<z<2$ для всех представленных распределений МГД величин. Расчет течения проведен для следующих параметров задачи: $n_{o}=10^{15} \mathrm{~cm}^{-3}, T_{o}=2$ эВ, $J_{p}=300 \mathrm{\kappa A}, L=60 \mathrm{~cm}, R_{o}=20 \mathrm{cм}$, которые в рассматриваемом случае дейтерий-тритиевой плазмы приводят к значениям $\sigma_{o}=386$ и $\beta=0.009$. Масштаб векторов на рис.1в и рис.1г определяется значениями $H_{*}=0.3$ и $V_{*}=1.5 \cdot V_{o}$, указанными на рисунках. Здесь единица скорости равна $V_{o}=1.3 \cdot 10^{7} \mathrm{~cm} / \mathrm{c}$. Следует напомнить, что в качестве единицы магнитного поля выступает характерное значение его азимутальной компоненты, определяемое разрядным током в ускорителе $H_{o}=2 J_{p} / c R_{o}$. В данном случае взаимодействие потока плазмы с магнитным полем соленоида рассматривается в отсутствие продольного магнитного поля в канале КСПУ. Соответственно на входе в канал $H_{z}^{o}=0$.

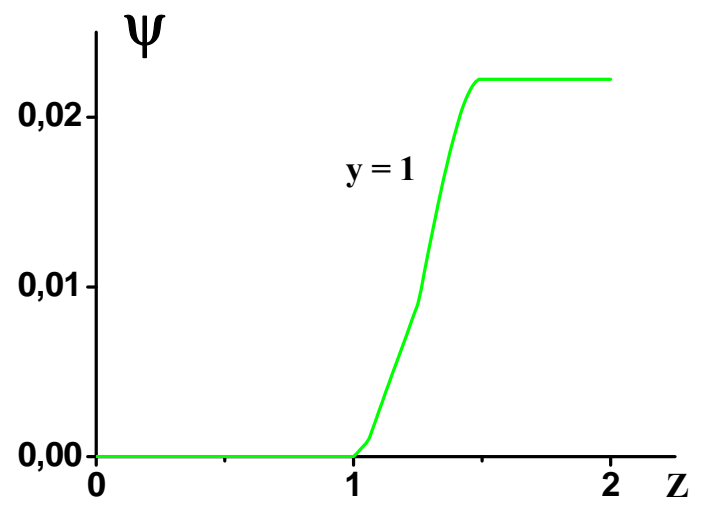

Рис.2. Распределение функции $\psi=r A_{\varphi}$ вдоль изолятора.

В наиболее узкой части канала происходит переход скорости потока через скорость быстрой магнитозвуковой волны или скорость сигнал [3] в формирующемся трансзвуковом течении. Распределения плотности и температуры на рис.1а и рис.1б показывают, что на выходе из профилированной части канала ускорителя в результате схождения сверхзвукового потока на оси системы формируется коническая ударная волна, структура которой сохраняется при значениях $1<z<1.4$, т.е. до взаимодействия потока с магнитным полем соленоида. На конической ударной волне происходит также излом линий потока, наблюдаемый на рис.1в. Считаем, что внешний электрод является анодом. Этому соответствует направление электрического тока (линии уровня функции $r H_{\varphi}=$ const), представленное на рис.1г.

Магнитное поле, создаваемое кольцевыми проводниками с током, проникает в поток плазмы в соответствии с рис.1г, но оно не проникает на всю глубину объема плазмы для реальных значений магнитной вязкости. Выра- 
женного ударного перехода на входе в соленоид, который мог бы сформироваться под действием магнитной преграды, не наблюдается для рассматриваемых небольших токов в кольцевых проводниках. Магнитное поле кольцевых проводников внутри соленоида оказывает влияние на исследуемые высокоскоростные потоки плазмы только во внешней области, периферийной по отношению к большей части основного потока для заданного значения тока в катушках. Сильное магнитное поле в окрестности внешней границы выталкивает плазму в силу ее диамагнетизма. Это приводит к значительному разрежению в окрестности внешней границы на всем протяжении соленоида при $1.25<z<2$. На рис.1а этому отвечает протяженная область разрежения в окрестности изолятора. Здесь же наблюдается вращение разреженной плазмы, представленное на рис.1в, на котором белый фон отвечает нулевым значениям азимутальной скорости вращения. В данном случае вращение локализовано у поверхности изолятора, т.к. в основном потоке плазмы отсутствует продольное магнитное поле, которое также привело бы к вращению. При вхождении потока в область магнитного поля соленоида меняется прежняя структуру течения, связанная с конической ударной волной, которая в результате деформируется.

Для рассматриваемого варианта с кольцевыми проводниками, образующими соленоид, на рис.3 представлены соответствующие одномерные графики зависимости ряда МГД переменных вдоль различных координатных линий, определяемых значением $y$ в соотношении (9). Рис.3а, отвечающий продольному магнитному полю $H_{z}$ в безразмерных переменных для значений $y=0.8$ и $y=0.5$, также иллюстрирует сужение области проникновения магнитного поля кольцевых проводников в поток плазмы по мере удаления от внешней границы с максимальными значениями продольного магнитного поля. Изменение концентрации представлено на рис.3б для тех же значений $y=0.8$ и $y=0.5$. Наблюдается относительно небольшое увеличение плотности перед областью сильного магнитного поля на входе в соленоид.

Рис.3в показывает, как меняется распределение температуры при $y=0$ и $y=0.5$ в процессе инжекции потока плазмы в область магнитного поля, создаваемого соленоидом. Резкое увеличение значений температуры в окрестности оси системы при $y=0$ и $z \approx 1$ отвечает области компрессии за срезом внутреннего электрода. Распределение температуры вдоль средней координатной линии для $y=5$ показывает, что инжекция потока в магнитное поле соленоида также сопровождается увеличением температуры при $z \approx 1.5$. Распределения температуры на рис.1б и рис.3в для значений $y=0$ и $z \sim 1.5$ говорят о том, что обтекание области сильного магнитного поля приводит к частичной трансформации и преобразованию кинетической энергии в теп- 
ловую или внутреннюю энергию плазмы в окрестности оси системы. Следствием этой трансформации является локальный максимум в распределении температуры, наблюдаемый на рисунках для значений $z \sim 1.5$ в окрестности оси. Однако далее в пределах соленоида не наблюдается увеличения температуры, поскольку отсутствует механизм нагрева плазмы, выявленный для случая кольцевых проводников, находящихся на определенном расстоянии друг от друга [2].

a)

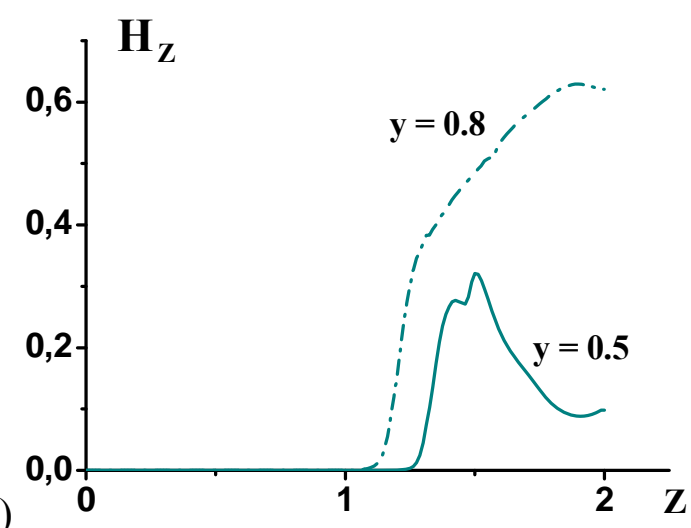

в)

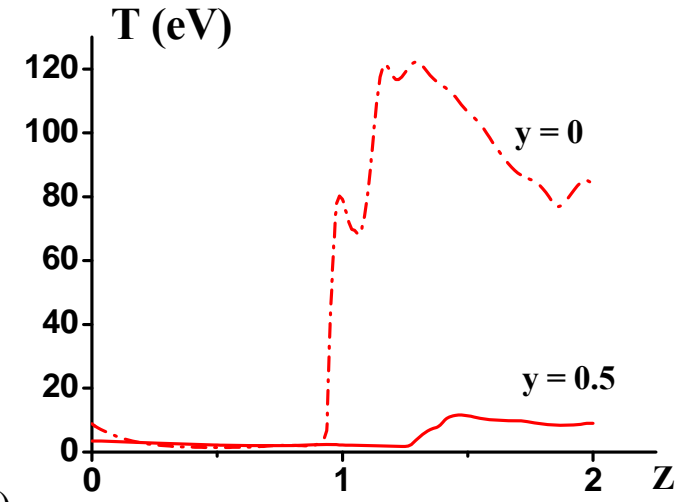

б)

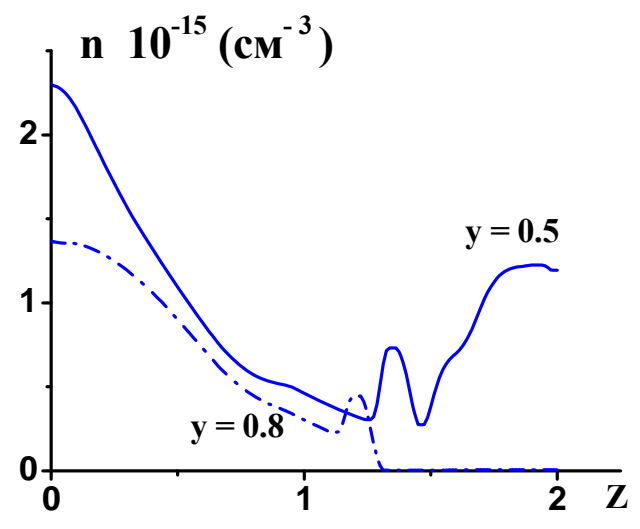

г)

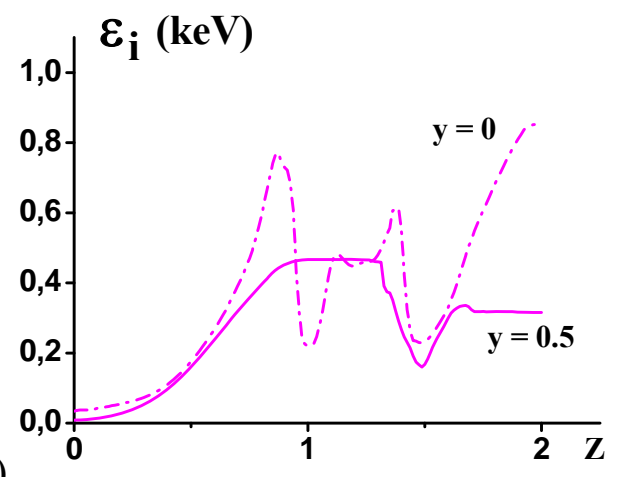

Рис.3. Значения МГД переменных вдоль различных координатных линий при инжекции потока плазмы в магнитное поле соленоида.

Одной из важных характеристик плазменных ускорителей является энергия ионов $\varepsilon_{i}=m_{i} V_{i}^{2} / 2$ на выходе. На рис.3б изображены соответствующие одномерные графики зависимости энергии ионов $\varepsilon_{i}$ вдоль средней координатной линии для $y=0.5$, а также вдоль линии $y=0$, которые определяются соотношением (9). Ускорение плазмы происходит в пределах соплообразной части канала при $0<z \leq 1$. При этом наблюдается определенная зависимость ускорения различных слоев плазмы в зависимости от их удаления от оси системы при $0<z \leq 1$. Особенности распределения при $y=0$ и $z \approx 1$ связаны с резким уменьшением скорости и энергии ионов в области компрессии, расположенной за внутренним электродом. Резкое изменение энергии ионов при $y=0.5$ и $z \approx 1.4$ вызвано торможением потока магнитным полем на 
входе в соленоид. Изменение энергии в окрестности оси при $y=0$ и $z \approx 1.5$ также обусловлено торможением потока, но после обтекания области сильного магнитного поля и схождения потока на оси системы. Здесь наблюдается локальный минимум в распределении энергии ионов.

Расчеты также показали, что увеличение тока в кольцевых проводниках приводит к тому, что более сильное магнитное поле проводников с током проникает в плазму на большую глубину. В этом случае обтекание плазмой более обширной области сильного магнитного поля приводит к более значительной трансформации кинетической энергии в тепловую или внутреннюю энергию плазмы в окрестности оси системы при значениях $z \sim 1.5$ на входе в соленоид. Здесь наблюдается существенный рост значений температуры. С увеличением тока в кольцевых проводниках происходит также более значительная деформация прежней структуры течения, связанной с конической ударной волной.

Распределение плотности энергии излучения $U$ представлено на рис.4 линиями уровня функции, соответствующей безразмерному значению $c U$. Векторное поле на данном рисунке отвечает плотности потока энергии излучения $\mathbf{W}$. Поле излучения соответствует распределениям плотности и температуры, изображенным на рис.1. Перенос излучения рассчитан в рамках локального термодинамического равновесия с учетом соотношений (2),(3). Безразмерная величина $\tilde{U}$, представленная на рис.4, связана с плотностью энергии излучения $U$ соотношением $\tilde{U}=10^{6} \cdot c \cdot U / W_{o}$, где единица плотности потока энергии излучения равна $W_{o}=V_{o} H_{o}{ }^{2} / 4 \pi=1.14 \cdot 10^{13}$ эрг $/ \mathrm{cm}^{2}$ сек для указанных выше исходных параметров $n_{o}, T_{o}, J_{p}$ и $L$. Масштаб векторов $\mathbf{W}$ определяется модулем вектора $W_{*}=10^{-6} \cdot W_{o}$, который указан на рис.4.

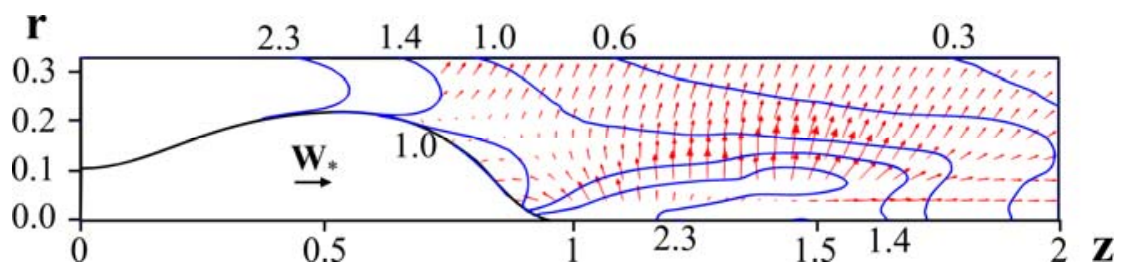

Рис.4. Распределение плотности энергии излучения $U$ и векторное поле плотности потока энергии излучения $\mathbf{W}$ в потоке плазмы, инжектируемой в соленоид.

Плотность энергии излучения $U$ имеет относительно большие значения в области компрессии и области вхождения потока в магнитное поле соленоида. Поток энергии излучения $\mathbf{W}$ направлен во все стороны от данных областей с относительно высокими значениями температуры. Соответственно эти области является мощным источником излучения. При этом основной вклад в общее поле излучения вносит рекомбинационная часть спектра. 
Модель переноса излучения, представленная подробно в [16,35], позволяет определить спектр излучения в направлении любого луча, выходящего из объема плазмы, в любом диапазоне частот. Это открывает новые возможности для сопоставления с экспериментальными данными и проведения комплексных расчетных и экспериментальных исследований на установках КСПУ, которые являются многофункциональными системами с широким кругом приложений.

\section{5. Заключение}

Численное исследование инжекции высокоскоростного потока достаточно плотной плазмы в магнитное поле кольцевых проводников с током, образующих соленоид, проведено на основе модифицированной системы МГД уравнений, представленных в терминах векторного потенциала магнитного поля с учетом электропроводности и теплопроводности среды, а также переноса излучения. Разработанная модель позволила изучить динамику плазмы в системе, состоящей из плазменного ускорителя, используемого для генерации потоков, и соленоида, расположенного на одной оси с КСПУ. Выявлены основные закономерности, возникающие в процессе инжекции осесимметричного потока плазмы в магнитное поле соленоида. Показано, что в объеме соленоида небольшие по величине токи в кольцевых проводниках создают магнитное поле, которое не оказывает значительного влияния на основной поток, одновременно изолируя его от катушек с током в результате образования слоя разреженной плазмы между катушками и высокоскоростным потоком плотной плазмы. При этом создается своего рода канал для транспортировки плазмы. Внутри соленоида отсутствует механизм для нагрева плазмы, и ее течение в пределах соленоида не сопровождается увеличением температуры среды. Определены интегральные характеристики излучения в формирующемся потоке плазмы.

\section{СПИСОК ЛИТЕРАТУРЫ}

1. А.Н. Козлов. МГД модель взаимодействия потока плазмы в КСПУ с магнитным полем кольцевого проводника с током // Физика плазмы, 2019, т.45, №2, с.160-171; A.N. Kozlov. MHD model of plasma flow interaction in the QSPA with a magnetic field of ring conductor with a current // Plasma Physics Reports, 2019, v.45, №2, p.147-158.

2. A.N. Kozlov. The study of high-velocity flow injection into the set of magnetic field coils coupled to plasma accelerator // Plasma Physics and Controlled Fusion, 2019, v.61, №3, Ar. 035008.

3. А.И. Морозов. Введение в плазмодинамику. - М.: Физматлит, 2008, 613 с.; A.I. Morozov. Vvedenie v plazmodinamiku. - M.: Fizmatlit, 2008, 613 s.

4. К.В. Брушлинский, А.М. Заборов, А.Н. Козлов, А.И. Морозов, В.В. Савельев. Численное 
моделирование течений плазмы в КСПУ // Физика плазмы, 1990, т.16, №2, с.147-157. K.V. Brushlinskii, A.M. Zaborov, A.N. Kozlov, A.I.Morozov, V.V. Savelyev. Numerical simulation of plasma flows in the QSPA // Sov. J. Plasma Phys., 1990, v.16, p.79-89.

5. К.В. Брушлинский. Математические основы вычислительной механики жидкости, газа и плазмы. - Долгопрудный: Издат. Дом "Интеллект", 2017.

K.V. Brushlinskii. Mathematical Foundations of Liquid, Gas, and Plasma Computational Mechanics. - Dolgoprudnyi: Intellekt, 2017.

6. N. Klimov, V. Podkovyrov, A. Zhitlukhin, D. Kovalenko, et al. Experimental study of PFCs erosion under ITER-like transient loads at plasma gun facility QSPA // Journal of Nuclear Materials, 2009, v.390-391, p.721-726.

7. I.E. Garkusha, V.V. Chebotarev, S.S. Herashchenko, V.A. Makhlaj, et al. Novel test-bed facility for PSI issues in fusion reactor conditions on the base of next generation QSPA plasma accelerator // Nuclear Fusion, 2017, v.57, №11, Ar. 116011.

8. V.M. Astashynski, S.I. Ananin, et al. Materials surface modification using quasi-stationary plasma accelerators // J. Surface and Coating Technology, 2004, v.180-181, p.392-395.

9. А.Н. Козлов. Влияние продольного магнитного поля на эффект Холла в канале плазменного ускорителя // Известия РАН, МЖГ, 2003, №4, с.165-175;

A.N. Kozlov. Influence of a longitudinal magnetic field on the Hall effect in the plasma accelerator channel // Fluid Dynamics, 2003, v.38, p.653-661.

10. A.N. Kozlov. Basis of the quasi-steady plasma accelerator theory in the presence of a longitudinal magnetic field // J. Plasma Physics, 2008, v.74, №2, p.261-286.

11. А.Н. Козлов. Двухжидкостная магнитогидродинамическая модель течений плазмы в квазистационарном ускорителе с продольным магнитным полем // Прикладная механика и техническая физика, 2009, т.50, №3, с.44-55;

A.N. Kozlov. Two-fluid magneto hydrodynamic model of plasma flows in a quasi-steadystate plasma accelerator with a longitudinal magnetic field // Journal of Applied Mechanics and Technical Physics, 2009, v.50, №3, p.396-405.

12. А.Н. Козлов. Исследование приэлектродных процессов в квазистационарных плазменных ускорителях с непроницаемыми электродами // Физика плазмы, 2012, т.38, №1, c.15-25.

A.N. Kozlov. Study of the near-electrode processes in quasi-steady plasma accelerators with impenetrable electrodes // Plasma Physics Reports, 2012, v.38, p.12-21.

13. К.В. Брушлинский, Н.С. Жданова, Е.В. Степин. Ускорение плазмы в коаксиальных каналах с профилированными электродами и продольным магнитным полем // Журнал вычислительной математики и математической физики, 2018, т.58, №4, с.607-617; K.V. Brushlinskii, N.S. Zhdanova, E.V. Stepin. Acceleration of plasma in coaxial channels with preshaped electrodes and longitudinal magnetic field // Computational Mathematics and Mathematical Physics, 2018, v.44, №4, p.593-603.

14. A.N. Kozlov. The study of plasma flows in accelerators with thermonuclear parameters // Plasma Physics and Controlled Fusion, 2017, v.59, №11, Ar. 115004.

15. А.И. Морозов, А.Н. Козлов. Эффект самоочищения потока водородной плазмы в ускорителе КСПУ // Физика экстремальных состояний вещества. Под ред. Фортова В.Е. и др. Изд. ИПХФ РАН, Черноголовка, 2007, с.316-319;

A.I. Morozov, A.N. Kozlov. Self-cleaning effect of hydrogen plasma flow in the QSPA accelerator // Physics of Extreme States of Matter, Ed. by V. E. Fortov et al. Chernogolovka: IPKhF RAN, 2007, p.316-319.

16. A.N. Kozlov, V.S. Konovalov. Numerical study of the ionization process and radiation 
transport in the channel of plasma accelerator // Communications in Nonlinear Science and Numerical Simulation (CNSNS), 2017, v.51, p.169-179.

17. А.Н. Козлов. Кинетика ионизации и рекомбинации в канале плазменного ускорителя // Известия РАН.,МЖГ, 2000, №5, с.181-188;

A.N. Kozlov. Ionization and recombination kinetics in a plasma accelerator channel // Fluid Dynamics, 2000, v.35, p.784-790.

18. А.А. Бармин, А.Н. Козлов. Структура стационарного фронта ионизации в канале плазменного ускорителя // Известия РАН, МЖГ, 2013, №4, с.155-167;

A.A. Barmin, A.N. Kozlov. Structure of a steady-state ionization front in the plasma accelerator channel // Fluid Dynamics, 2013, v.48, p.556-566.

19. А.И. Морозов, В.В. Савельев. О галатеях-ловушках с погруженными в плазму проводниками // Успехи физических наук, 1998, т.168, №11, с.1153-1194;

A.I. Morozov, V.V. Savelyev. On galateas-magnetic traps with plasma-embedded conductors // Physics-Uspekhi (Advances in Physical Sciences), 1998, v.41, p.1049-1089.

20. A.I. Morozov, A.I. Bugrova, A.M. Bishaev, M.V. Kozintseva, A.S. Lipatov. Plasma parameters in the upgraded Trimyx-M Galathea // Technical Physics, 2007, v.52, p.1546-1551.

21. К.В. Брушилнский, А.С. Гольдич, Н.А. Давыдова. Плазменные конфигурации в ловушках-галатеях и токовых слоях // Математическое моделирование, 2016, т.28, №7, c. 107-120;

K.V. Brushlinsky, A.S. Goldich, N.A. Davydova. Plasma Configurations in Galatheya Traps and Current Sheets // Mathematical Models and Computer Simulations, 2017, v.9, №1, p.60-70.

22. A.V. Burdakov, V.V. Postupaev. Multiple-mirror trap: a path from Budker magnetic mirrors to linear fusion reactor // Physics-Uspekhi (Advances in Physical Sciences), 2018, v.61, p.651-671.

23. В.В. Савельев. Динамика плазменной струи в магнитном поле квадрупольного типа. - М.: ИПМ им. М.В. Келдыша РАН, 1991, препринт №117.

V.V. Savelev. Dinamika plazmennoi strui v magnitnom pole kvadrupolnogo tipa. - M.: IPM im. M.V. Keldysha RAN, 1991, preprint №117.

24. Л.Д. Ландау, Е.М. Лифиии. Электродинамика сплошных сред. - М.: Наука, 2-е изд., 1982, 620 c.;

L.D. Landau, E.M. Lifshits. Elektrodinamika sploshnykh sred. - M.: Nauka, 2-e izd., 1982, $620 \mathrm{~s}$.

25. А.Г. Куликовский, Г.А. Любимов. Магнитная гидродинамика. 2-е изд. - М.: Логос, 2005, 328 c.;

A.G. Kulikovskii, G.A. Liubimov. Magnitnaia gidrodinamika. 2-e izd. - M.: Logos, 2005, $328 \mathrm{~s}$.

26. С.И. Брагинский. Явление переноса в плазме. // Вопросы теории плазмы. Под ред. М.А. Леонтовича. М.: Госатомиздат, 1963. вып.1, с.183-272;

S.I. Braginsky. Transport processes in a plasma. - NY: Consultants Bureau, Reviews of Plasma Physics, 1965, v.1, p.201-292.

27. Н. Кролл, А. Трайвелпис. Основы физики плазмы. - М.: Мир, 1975, 525 с.;

N.A. Krall, A.W. Trivelpiece. Principles of plasma physics. - NY: McGraw-Hill, 1978.

28. Я.Б. Зельдович, Ю.П. Райзер. Физика ударных волн и высокотемпературных гидродинамических явлений. - М.: Наука, 1966, 686 с.;

Y.B. Zel'dovich, Y.P. Raizer. Physics of shock waves and high-temperature hydrodynamic phenomena. - NY: Dover Publication Inc. Mineola, 2002. 
29. Д. Михалас. Звездные атмосферы. 1 часть. - М.: Мир, 1982, 352 с.;

D. Mihalas. Stellar atmospheres. - San Francisco: W. H. Freeman, 1978.

30. Б.Н. Четверушкин. Математическое моделирование задач динамики излучающего газа. - М.: Наука, 1985, 304 с.;

B.N. Chetverushkin. Matematicheskoe modelirovanie zadach dinamiki izluchaiushchego gaza. - M.: Nauka, 1985.

31. А.Ф. Никифоров, В.Г. Новиков, В.Б. Уваров. Квантово-статистические модели высокотемпературной плазмы. - М.: Физматлит, 2000, 399 с.;

A.F. Nikiforov, V.G. Novikov, V.B. Uvarov. Quantum-statistical models of hot dense matter. Methods for computation opacity and equation of state. - Switzerland, Basel: Birkhauser Verlag, 2005.

32. J.I. Castor. Lectures on radiation hydrodynamics. Livermore: Lawrence Livermore National Laboratory, 2000.

33. B.A. Гасилов и др. Пакет прикладных программ MARPLE3D для моделирования на высокопроизводительных ЭВМ импульсной магнитоускоренной плазмы // Математическое моделирование, 2012, т 24, №1, с 55-87;

B.A. Gasilov $i d r$. Paket prikladnykh programm MARPLE3D dlia modelirovaniia na vysokoproizvoditelnykh EVM impulsnoi magnitouskorennoi plazmy // Matematicheskoe modelirovanie, 2012, t 24, №1, s 55-87.

34. A.N. Kozlov, I.E. Garkusha, V.S. Konovalov, V.G. Novikov. The radiation intensity of the Lyman alpha line at the ionization front in the quasi-steady plasma accelerator // Problems of Atomic Science and Technology. Series: Plasma Physics, 2013, №1, p.128-130.

35. А.Н. Козлов, В.С. Коновалов. 3D модель переноса излучения в потоках ионизующегося газа и плазмы. - М.: ИПМ им. М.В. Келдыша РАН, 2016, препринт №86.;

A.N. Kozlov, V.S. Konovalov. 3D model of the radiation transport in flows of the ionizing gas and plasma. - M.: KIAM RAS, 2016, preprint №86.

36. Э. Оран, Дж. Борис. Численное моделирование реагирующих потоков. - М.: Мир, 1990, 661 c.;

E.S. Oran, J.P. Boris. Numerical simulation of reactive flow. - NY: Elsevier, 1987.

37. Л.М. Дегтярев, А.П. Фаворский. Потоковый вариант метода прогонки для разностных задач с сильно меняющимися коэффициентами // Журнал вычислительной математики и математической физики, 1969, т.9, №1, с.211-218;

L.M. Degtyarev, F.P. Favorskii. Flow variant of the sweep method for difference problems with strongly varying coefficients // Sov. J. Computational Mathematics and Mathematical Physics, 1969, v.9, p.285-294.

38. А.Н. Козлов. Динамика вращающихся потоков в канале плазменного ускорителя с продольным магнитным полем // Физика плазмы, 2006, т.32, №5, с.413-422;

A.N. Kozlov. Dynamics of rotating flows in plasma accelerator channels with a longitudinal magnetic field // Plasma Physics Reports, 2006, v.32, p.378-387.

Поступила в редакцию 14.10.2019

После доработки 14.10.2019

Принята к публикации 25.11.2019 